\title{
FLORENCE GAUZY
}

\section{DIE VERTEIDIGUNG: VON DER ANPASSUNG DER DOKTRINEN ZU GEMEINSAMEN KONZEPTEN?}

In einem kürzlich erschienenen Artikel über den Élysée-Vertrag bittet Wolfram Vogel die Akteure und Spezialisten der deutsch-französischen Kooperation, das Pathos endlich in die Regale der Erinnerung zu verbannen. ${ }^{1}$ Sicherlich, so erläutert er, verlangte der symbolische Charakter der von Bundeskanzler Adenauer und General de Gaulle im Namen der "Erbfeinde« besiegelten Aussöhnung geradezu nach einer würdevollen Feier mit Pomp und Gloria. Dazu paßte zweifellos auch bestens ein Vertrag, selbst wenn er anfangs in dieser Form nicht vorgesehen war, ${ }^{2}$ aber seit 1963 hätten sich die Hüter des Vertrages unter reichlicher Zuhilfenahme von gemeinsamen Erklärungen, Freundschaftsbezeugungen und Kommuniqués, die von guten Absichten nur so strotzten, auf ein riskantes gegenseitiges Wettrennen eingelassen, das in der Öffentlichkeit den Eindruck eines fiktiven Erfolges hervorruft, der die tatsächliche Einschätzung der Lage aber verfälscht. ${ }^{3}$

Über 40 Jahre nach Unterzeichnung des Vertrages ist dieser Appell nicht unangebracht, insbesondere im Bereich der Verteidigung. Da ständig die deutsch-französische Kooperation zelebriert wird, beginnt die öffentliche Meinung, sofern sie ihrer denn nicht schon überdrüssig ist, wie es für die junge Generation bereits zuzutreffen scheint, schließlich auch an ihren Erfolg zu glauben. In dem einen wie dem anderen Fall hält sie die Kooperation für ganz normal. Aber sie ist es keineswegs und sie ist es niemals gewesen. Um das zu verstehen, reicht ein Blick auf die Entstehungsphase und die ersten Jahre des

1 Wolfram VOGEL, Der Élysée-Vertrag. Die Zukunftsfähigkeit eines historischen acquis: Fünf Thesen, in: Dokumente, 58 (2002) 6, S. 13-16; DERS., Die Zukunft des ÉlyséeVertrags, in: Blätter für deutsche und internationale Politik, 1 (2003), S. 13-16.

2 Hans-Peter SCHWARz, Präsident de Gaulle, Bundeskanzler Adenauer und die Entstehung des Élysée-Vertrages, in: Wilfried LOTH, Robert PICHT (Hg.), De Gaulle, Deutschland und Europa, Opladen 1991, S. 169-179.

3 Es gibt ein Ritual während der deutsch-französischen Gipfeltreffen, das Pierre Jardin sehr gut beschrieben hat: Pierre JARDIN, Die deutsch-französischen Gipfeltreffen seit 1963, in: Centre d'Études d'histoire de la Défense (CEHD), Centre d'Études en Sciences Sociales de la Défense (C2SD) ( $\mathrm{Hg}$.), Bilan et perspectives de la coopération militaire francoallemande de 1963 à nos jours, Paris 1999, S. 41-69. 
Vertrages. ${ }^{4}$ Es gab nichts Normales in der deutsch-französischen Annäherung, und es brauchte ein großes $\mathrm{Maß}$ an gutem Willen und Durchhaltevermögen der Akteure, ob sie nun zur Regierung gehörten oder nicht, um dem Vertrag Leben einzuhauchen: immerhin mehr als zwei Jahrzehnte.

Vor diesem doch recht trügerischen Erwartungshorizont kann man sich die Enttäuschung der Öffentlichkeit vorstellen, wenn sie wie im Vorfeld der Feierlichkeiten zum 40. Jahrestag des Vertrages feststellen muß, daß die Realität nicht immer den Hoffnungen gerecht wird. Daß Frankreich und Deutschland nicht von vornherein eine gemeinsame Position in bezug auf eine militärische Intervention des Westens im Irak hatten, daß sie nicht die gleiche Vorstellung von der zu bildenden "Festung Europa" haben, daß das Eurocorps, die deutsch-französische Initiative schlechthin, die nach Ansicht ihrer Gründerväter Deutschland, Frankreich und ihre europäischen Partner mit einer autonomen militärischen Streitmacht versehen soll, kein Wundermittel ist und daB diese seit 1995 einsatzbereite gemischte Einheit, die ohne die Hilfe der NATO im Bereich des Lufttransports, der strategischen Aufklärung und im Kommandobereich, noch immer nicht in der Lage ist, eine Operation in einem modernen Krieg durchzuführen, all das ist Realität und mag einen traurig stimmen. Gleichwohl rechtfertigen diese Umstände keineswegs die übertrieben abwertenden Kommentare, die oft zu hören sind.

Denn trotz alledem läßt sich ein Ergebnis vorweisen, ein Ergebnis, das vielleicht nicht so ausgefallen ist, wie es sich die Unterzeichner gewünscht haben, aber ein Ergebnis immerhin. Und gerade dieses sollten die offiziellen Feiern in Erinnerung rufen, statt weitere Zukunftsperspektiven aufzuzeigen, die sich schon in der Vergangenheit wiederholt als sehr riskant erwiesen haben. Nehmen wir die bereits oben erwähnten Beispiele. Das kategorische "Nein" von Bundeskanzler Schröder zu einer Intervention im Irak, vermutlich aus wahltaktischen Erwägungen, war sicherlich einschneidend. Seine Weigerung hat dennoch nicht den Prozeß der deutsch-französischen Konsultationen behindert. Besser noch, im Gegenzug unterstützte Deutschland offen die französische Initiative im Sicherheitsrat der Vereinten Nationen, die darauf abzielte, den Grundprinzipien des internationalen Rechtes gegen die Idee eines Präventivkrieges durch die Annahme einer Resolution, die die Wiederaufnahme der Inspektionen im Irak forderte, Geltung zu verschaffen. Seit Ende der 1990er Jahre nehmen die europäischen Initiativen im Bereich der Verteidigung trotz unterschiedlicher deutsch-französischer Konzepte in zwei entscheidenden Punkten Formen an: einerseits über das Ausmaß der strategischen

4 Jacques BARIÉTY, De Gaulle, Adenauer et la genèse du traité de l'Élysée du 22 janvier 1963, in: Institut Charles de Gaulle (Hg.), De Gaulle en son siècle, Bd. 5: L'Europe, Paris 1992, S. 355-362. 
Autonomie Europas gegenüber der NATO; andererseits über die Bedeutung der militärischen Macht im Rahmen der zukünftigen Sicherheitspolitik. Auf der Basis des gemeinsamen deutsch-französischen Sicherheits- und Verteidigungskonzepts (1996) haben sich Deutschland und Frankreich im Juni 1999 im EU-Ministerrat in Köln verpflichtet, zivile und militärische Kapazitäten bereit zu stellen, die es der Europäischen Union ermöglichen, einen wichtigen Part in der Behandlung von Krisen und in der Verhütung von Konflikten auf internationaler Ebene zu spielen. Die Vereinigten Staaten haben sich im übrigen keinesfalls täuschen lassen und sofort auf die Ankündigung einer europäischen schnellen Eingreiftruppe von 60000 Mann reagiert - die innerhalb von 60 Tagen aufgestellt und in der Lage sein sollte, ein Jahr lang einen Auftrag vom Typ »Petersberg “ durchzuführen ${ }^{5}$ - nämlich durch eine ähnliche Gründung im Rahmen der NATO. Auf der Ebene des Eurocorps liegt der Erfolg in der Fähigkeit, gemeinsam in Krisensituationen eingreifen zu können dank einer verbesserten Interoperabilität der Soldaten, der Einheiten und der Bewaffnung, die von den Experten während der Einsätze des Eurocorps im Rahmen der KFOR und der SFOR in Bosnien-Herzegowina und im Kosovo gelobt wurde. Daß das Eurocorps selbst nicht in der Lage ist, Probleme zu lösen, deren Bewältigung größere finanzielle, menschliche und technologische Mittel erfordern, als die Mitgliedsländer im Rahmen ihres eigenen Verteidigungshaushaltes vorgesehen haben, ist nicht erstaunlich. Das Eurocorps ist nicht die NATO. Es ist nur ein Instrument im Dienste einer europäischen Sicherheitsund Verteidigungspolitik (ESVP), die noch zu definieren ist. Es ist trotzdem sehr nützlich. Seine Anpassungs- und Ausbaufähigkeit nach dem Prinzip der "combined joint task forces « (CJTF) ${ }^{6}$ könnte es zu einem wertvollen Instrument in einem neuen strategischen Kontext werden lassen, der durch die At-

5 Unter einem Auftrag vom Typ "Petersberg" versteht man ein großes Spektrum an Missionen von unterschiedlicher militärischer Intensität, die zur Aufrechterhaltung und Wiederherstellung des Friedens dienen: humanitäre Missionen oder die Evakuierung von Staatsbürgern, Friedensmissionen, Missionen von Kampftruppen zur Bewältigung von Krisensituationen, einschlieBlich der Wiederherstellung des Friedens. Das Dokument, auf das Bezug genommen wird, ist die Erklärung des Ministerrates der WEU von 1992. Der Vertrag von Amsterdam von 1997 übemimmt diese Definition und macht sie zum Ziel einer neuen europäischen Politik der Sicherheit und Verteidigung (ESVP).

6 Ein Konzept von anpassungsfähigen trennbaren, aber nicht getrennten Eingreiftruppen aller Waffengattungen, das im Rahmen der NATO 1996 entwickeit wurde und damals den gemeinsamen Einsatz der Truppen von NATO und WEU in Fragen der Verteidigung Europas vorsah. Der Einsatz der Truppen sollte entweder unter der Führung der NATO oder der WEU stattfinden oder aber unter dem gemeinsamen Kommando von beiden. Seit die EU allein für die Operationen der WEU verantwortlich ist, hängt die Anwendung des neuen militärischen Konzeptes von seiner Fähigkeit ab, Bedingungen einer reellen Interoperabilität mit den Strukturen der NATO zu schaffen; vgl. Johannes VARWICK, Sicherheit und Integration in Europa, Opladen 1998, S. 229-246. 
tentate vom 11. September 2001 entstanden ist. Seither steht die Bildung von multilateralen Ad hoc-Truppen im Rahmen einer präzise definierten internationalen Polizeiaktion mehr denn je auf der Tagesordnung, auch wenn sich Frankreich angesichts der Bildung solcher Truppen noch zögerlich verhält.

Dies führt uns zu einer zweiten Vorbemerkung. In der jüngsten Geschichte der deutsch-französischen Beziehungen waren die Fortschritte ein Ergebnis des Kooperationswillens, der oft genug aus strategischen Notwendigkeiten resultierte und die USA als »dritte Partei« zu berücksichtigen hatte. Auf jeder Etappe der Annäherung war es der Wille zur Kooperation der Beteiligten, der ausschlaggebend war und nicht der Umstand, ob nun gemeinsame Positionen vorhanden waren oder nicht. Er allein hat es beiden Ländern erlaubt, auf dem Wege der Kooperation voranzuschreiten und bei gleicher Gelegenheit auch die europäische Integration über alle unterschiedlichen Standpunkte hinweg vorwärts zu bringen. Im übrigen, um eine Binsenwahrheit aufzugreifen, die Bundeskanzler Ludwig Erhard so am Herzen lag, wenn in den Bereichen wie der Außen- und der Verteidigungspolitik die Standpunkte Deutschlands und Frankreichs nicht so weit auseinander gelegen hätten, wäre der Vertrag auch nicht nötig gewesen. ${ }^{7}$ Unter diesem Gesichtspunkt sind die Gültigkeitsbereiche recht bezeichnend. Die Betonung liegt auf den sensiblen Bereichen, während die Ebenen, auf denen eine breite Kooperation eher einfach zu erreichen war (z.B. die Wirtschaft) ausgeschlossen wurden. ${ }^{8}$ Diese beiden Punkte sind im Gedächtnis zu behalten, wenn eine Bilanz der deutsch-französischen militärischen Zusammenarbeit seit 1963 zu ziehen sein wird.

\section{Ein Wendepunkt in der Geschichte der deutsch-französischen Militärkooperation?}

Bedeutete der Vertrag einen Wendepunkt in der Geschichte der deutschfranzösischen Militärkooperation? Ja und nein. Die deutsch-französische Militärkooperation setzte nicht erst mit dem Vertrag ein, denn 1963 sind die Stabschefs beider Länder schon seit mehr als zehn Jahren gewohnt zusammenzuarbeiten, was auf ihre Beteiligung am gescheiterten EVG-Projekt und

7 Ludwig Erhard am 12.6.1965: „Wir brauchen ja keinen Freundschaftsvertrag, wenn wir von vorneherein in allen Fragen einer Meinung wären. Der Freundschaftsvertrag ist ja gerade dazu da, um auch unterschiedliche Auffassungen von Mal zu Mal stärker sich einander annähern zu lassen. « Bulletin der Bundesregierung Nr. 102 vom 15.6.1965, S. 817.

8 Siehe dazu den Beitrag von Werner Bührer in diesem Band. 
ihre gemeinsame Arbeit in WEU und NATO nach der Aufnahme der Bundesrepublik in diese beiden Organe der internationalen Verteidigung zurückgeht. Außerdem hatte die französische Regierung über den bereits bestehenden Rahmen hinaus sehr früh nach der Unterzeichnung der Pariser Verträge (1954) zum Ausdruck gebracht, daß sie an einem verstärkten bilateralen militärischen Dialog interessiert sei, insbesondere in Rüstungsfragen. $\mathrm{Zu}$ diesem Zeitpunkt war Paris über die Entstehung einer zivilen westdeutschen Nuklearindustrie beunruhigt und fürchtete, da $B$ diese in eine unkontrollierbare militärische Nutzung abgleiten und sich daraus eine enge Zusammenarbeit Deutschlands mit den Vereinigten Staaten und Großbritannien entwickeln könnte. Die Möglichkeit einer deutsch-französischen Zusammenarbeit bei Rüstungsproduktion und Strategieplanung kam somit schnell über den Status eines Gedankenspiels hinaus, und sei es auch nur, um nach bewährter Methode durch die eigene Teilnahme die bundesdeutsche Nuklearproduktion im Auge zu behalten. 1955 schlug die französische Regierung Deutschland vor, sich an der Entwicklung einer Fabrik zur isotopischen Spaltung zu beteiligen. Mehr als das industrielle Projekt überrascht seine strategische Dimension. Das Angebot Frankreichs umfaßte dreierlei: erstens die Anstrengungen in der Atomforschung auf mehrere Schultern zu verteilen; zweitens eine langfristige Zusammenarbeit im Bereich der Rüstung aufzubauen; und drittens die Ausarbeitung gemeinsamer Konzepte für die Planung und den Einsatz dieses Materials. Bonn zeigte sich zunächst sehr zurückhaltend. Sie hatte kommerzielle Kontakte zu den Vereinigten Staaten aufgebaut, von denen ihre Sicherheit abhing. So blieben die Ergebnisse dieser ersten französischen Annäherung eher bescheiden. Verwiesen sei auf die Gründung des deutsch-französischen Forschungsinstituts in Saint-Louis 1958 und die Unterzeichnung einiger Verträge über Waffenkäufe.

1956 veränderten sich die internationale Lage und die amerikanische $\mathrm{Nu}$ klearstrategie, die sich vom strikten Konzept der Abschreckung zu entfernen schien. Somit war es Frankreich möglich, sein Projekt einer deutschfranzösischen Nuklearkooperation in einem günstigeren Umfeld wieder aufzugreifen. Paris bot der Bundesrepublik »eine Zusammenarbeit im Bereich der Rüstung an, die sich auch auf die Strategieplanung und auf die Formulierung einer gemeinsamen Konzeption sowie auf die gemeinsame Offiziersausbildung « erstrecken sollte. ${ }^{9}$ Nach einer gewissen Zeit des Abwartens reagierte Bonn Ende 1956 positiv, nachdem Franz Josef Strauß in der Nachfolge von Theodor Blank zum Verteidigungsminister ernannt worden war. Aus diesem plötzlichen Umschwung und dieser Phase der großen strategischen Annäherung zwischen der Bundesrepublik und Frankreich - von allerdings recht kurallemands, 1954-1996, Paris 1996, S. 47. 
zer Dauer! - entstanden 1957 wichtige Abkommen: das deutsch-französische Abkommen von Colomb-Béchar und ein deutsch-französisch-italienisches Protokoll, das auf Initiative von Strauß und seinem französischen Amtskollegen, dem Gaullisten Jacques Chaban-Delmas, bereits die großen Linien einer tatsächlichen gemeinsamen europäischen Rüstungspolitik enthielt, ausgeweitet auch auf den Nuklearsektor (Standardisierung des Materials, gemeinsame rationalisierte Leitung der Forschungszentren und der industriellen Ressourcen etc.). Hier findet sich bereits die Idee, da $\mathrm{B}$ ein solches Programm nur sinnvoll durchgeführt werden könne, wenn eine gemeinsame militärische Konzeption (Organisation, Einsatzbedingungen und die Bewaffnung der nationalen Streitkräfte) vorhanden ist. ${ }^{10}$ Das Protokoll präzisierte, daß die drei Länder diese Kooperation innerhalb des bereits existierenden institutionellen Rahmens (NATO und WEU) einbetten sollten, aber unter Hinweis auf die Existenz spezifischer europäischer Probleme. Da dieses Protokoll nur die unterzeichnenden

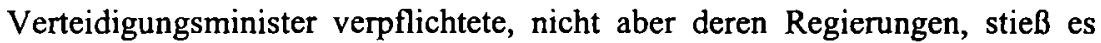
sehr schnell auf hohe Hindernisse. Es gab nämlich einen unvorhergesehenen Akteur in den Beziehungen zwischen diesen drei Ländern: die Vereinigten Staaten, gegenüber denen Bonn und Paris recht zwiespältige Positionen einnahmen, wobei jeder versuchte, der privilegierte Partner von Washington zu werden. Abgesehen von der direkten Umgebung von Strauß und Chaban ging niemand in den jeweiligen Regierungen in den Jahren 1956-1958 so weit wie die Formulierungen im Protokoll und niemand dachte ernsthaft an den Aufbau einer europäischen Nuklearmacht.

Der militärische Dialog zwischen Frankreich und Deutschland wurde mit der Rückkehr von Charles de Gaulle an die Macht und sein entscheidendes Treffen in Rambouillet mit Bundeskanzler Adenauer im Jahre 1960 auf einer neuen Grundlage wieder aufgenommen. Die bilateralen sogenannten StraußMessmer-Abkommen wurden im Oktober desselben Jahres unterzeichnet." Sie erlaubten es der Bundeswehr, in Frankreich Übungen abzuhalten und dort logistische Basen aufzubauen, in denen 50000 Tonnen Munition gelagert sowie schwere Gerätschaften instand gehalten werden konnten, während die deutsche Luftwaffe nun über Einrichtungen auf den französischen Flughäfen verfügte. ${ }^{12}$ Doch das war nicht alles, denn es bestand der gemeinsame Wille, eine militärische Zusammenarbeit zwischen diesen beiden Ländem außerhalb des Rahmens der NATO aufzubauen. Während auf internationaler Ebene ein

10 Ibid. S. 93-95.

1 Ibid. S. 157f.

12 Ulrich DE MAIZJĖRE, Die praktischen Folgen des Élysée-Vertrages im militärischen Bereich, in: Winfried LOTH, Robert PICHT (Hg.), De Gaulle, Deutschland und Europa, Opladen 1991, S. $203 f$. 
Wiederaufflammen des Kalten Krieges nach dem Scheitern des Gipfeltreffens der Großen Vier in Paris im Mai 1960 zu verzeichnen war, hegten Deutschland und Frankreich dieselbe Befürchtung: Die USA könnten ihre Nuklearwaffen aus Europa abziehen. Angesichts dessen, was als Schwächung der amerikanischen Sicherheitsgarantie für Europa empfunden wurde, die doch immerhin die Existenzberechtigung für die Allianz war, versuchten Paris und Bonn die existierenden bilateralen Bande enger zu knüpfen. Auf Bitte von Adenauer fanden auf Stabsebene deutsch-französische Gespräche über militärisch-strategische Fragen von gemeinsamem Interesse statt. Man sprach vor allem über die sowjetische Bedrohung, die amerikanische Strategie und die Notwendigkeit einer Vorneverteidigung. Diverse strategische Möglichkeiten wurden für die zentraleuropäische Bühne entwickelt. Eine sständige deutschfranzösische Stabsgruppe» wurde eingerichtet. Aus diesen Konsultationen sprach eine große Übereinstimmung bei der Frage eines Ersteinsatzes von Nuklearwaffen, über die Ablehnung, zwischen dem Einsatz von konventionellen und nuklearen Waffen eine Pause einzuhalten sowie über einen selektiven, begrenzten Einsatz von Nuklearwaffen. Der Historiker Georges-Henri Soutou kam zu dem Schluß: „Man stand den neuen amerikanischen Konzeptionen diametral gegenüber, die man angesichts der Brutalität der sowjetischen Strategie für viel zu abstrakt hielt, und man war sich zwischen Frankreich und Deutschland einig über das strikte Konzept der nuklearen Abschreckung. ${ }^{13}$ Einigkeit herrschte ebenfalls über die Notwendigkeit, die Bundesrepublik so weit wie möglich im Osten zu verteidigen, was die Befriedigung einer ständigen westdeutschen Forderung bedeutete, auch wenn Frankreich immer noch dem Konzept der zwei Schlachten anhing, erst die Schlacht um Deutschland und dann die Schlacht um Frankreich. All das ging den von de Gaulle gewünschten Gang. Er forderte seit 1958 eine tiefgreifende Reform der Atlantischen Allianz, der er vorwarf, die europäischen Sicherheitsinteressen nicht genügend zu berücksichtigen. Nach seiner Vorstellung sollte das Prinzip der Integration zugunsten einer Rückkehr zu einer klassischen Allianz zwischen den Vereinigten Staaten, Großbritannien und einer großen Union der Staaten Kontinentaleuropas aufgegeben werden, in der Frankreich aufgrund seiner Nuklearwaffen eine herausragende Stellung einnehme würde. Adenauer schien anfangs seine Vorstellungen zu teilen, so daß dieser zweite deutschfranzösische strategische Dialog in wichtigen Fragen, darunter auch im Nuklearbereich, Fortschritte brachte und auf eine positive Entwicklung hoffen ließ, ${ }^{14}$

${ }^{13}$ Georges-Henri SouTou, L'arrière-plan stratégique du traité de l'Élysée, in: CEHD, C2SD (Anm. 3), S. 108.

14 Pierre JARDIN, Adolf KimMel (Hg.), Les relations franco-allemandes depuis 1963, Paris 2001, S. 19-45. 
was dann in der Folge aber nicht mehr der Fall sein sollte. Somit reihten sich die im Élysée-Vertrag fixierten Bestimmungen zur Verteidigung in die Kontinuität einer deutsch-französischen Annäherung ein, die zehn Jahre zuvor ihren Ursprung genommen hatte.

Die bisherigen Ausführungen verdeutlichen, daß die Sicherheitsfragen in der Entstehungsphase des Élysée-Vertrages (1958-1963) neben dem Willen zu einer grundsätzlichen politischen Aussöhnung eine Schlüsselrolle spielten. Was war nun der genaue Inhalt des Vertrages? ${ }^{15}$ Im Bereich der Verteidigung erhielt die deutsch-französische Militärkooperation durch die Institutionalisierung in Vertragsform eine neue verstärkt bilaterale und programmatische $\mathrm{Di}$ mension. Zur gleichen Zeit wurde sie aber grundsätzlich durch den Entschluß zu einer watlantischen « Präambel in Frage gestellt, die den Inhalt dermaßen entwertete, daß ihm ein großer Teil der Substanz genommen wurde. ${ }^{16}$

Ins Auge sticht der Ehrgeiz des Vertrages, der aus seinem Inhalt, seiner Organisation und seinen Zielen spricht. Der Vertrag sah vor, die Zusammenarbeit in einem Maße auszuweiten, daß praktisch die Grundlagen für eine deutschfranzösische Union gelegt wurden, zusammengeschweißt durch dieselben strategischen und taktischen Doktrinen, dieselben Kommandostrukturen, den Gebrauch desselben Materials. Die Führungsebenen der beiden Länder sollten ihre jeweiligen Doktrinen mit dem Ziel der Entwicklung von gemeinsamen Konzepten annähern - dies ist der Punkt, der uns besonders interessiert. Praktisch sollte diese Annäherung in den noch zu gründenden deutschfranzösischen Forschungsinstituten für operative Studien stattfinden. Die Basis bildete ein Austausch von Menschen auf allen Ebenen, von ganzen Einheiten bis zur Stabsebene, und dies sollte durch eine gemeinsame Arbeit im Bereich der Bewaffnung erleichtert werden, um letztendlich zur gemeinsamen Planung und Produktion von Waffen zu gelangen. Und schließlich sollten die Regierungen eine mögliche Ausweitung dieser Maßnahmen auf die zivile Verteidigung prüfen. Im Bereich der Organisation wurden regelmäßige Treffen der Verteidigungsminister beider Länder vereinbart, wenigstens alle drei Monate; der Stabschefs sogar alle zwei Monate. Sie sollten an der Arbeit der interministeriellen Kommission teilnehmen, die sich um die Umsetzung des Vertrages kümmern sollte. Das Ziel war klar definiert. Durch die Verstärkung der Zusammenarbeit zwischen diesen beiden Ländern sollte auf dem Weg zu einem geeinten Europa vorangeschritten werden. ${ }^{17}$ Welch ein Programm!

is Traité entre la République française et la République fédérale d'Allemagne sur la coopération franco-allemande, 22 janvier 1963, in: JARDIN, KIMMEL (Anm. 14), S. 53-57.

${ }_{16}$ Préambule à la loi de ratification adopté par le Bundestag, 16.5.1963, in: ibid. S. 76.

17 Ibid. S. 43. 
Von Anfang an stieß der Vertrag auf eine vehemente Ablehnung. Der Kontext, in den die Unterzeichnung fiel, einige Tage nach dem »Nein« von de Gaulle zum Beitritt Großbritanniens in die Europäische Wirtschaftsgemeinschaft, war wohl kaum von Vorteil. Das ist ein Punkt, den man hervorheben muB. Die Vereinigten Staaten, die dies alles sehr aufmerksam verfolgten, waren bereits vor seiner endgültigen Fertigstellung gegen den Vertrag - man erinnert sich noch an die Improvisation und die Aufregung, die der Vorbereitung des endgültigen Textes vorausging. ${ }^{18}$ Die Verkündung seiner Unterzeichnung am 22. Januar 1963 löste eine Schockwelle aus. Washington sah darin zu Unrecht eine weitere Herausforderung der amerikanischen Vormachtstellung und die Gefahr eines geheimen deutsch-französischen Nuklearabkommens. Auch in der Bundesrepublik war der Moment nicht gerade glücklich gewählt. Adenauer sah sich sowohl den ablehnenden Kräften im eigenen Lager als auch der sozialdemokratischen Opposition gegenüber, die dem deutsch-französischen Freundschaftsvertrag vorwarf, die atlantische Verankerung der Bundesrepublik zu gefährden und damit gleichzeitig auch die darauf basierende amerikanische Sicherheitsgarantie. Selbst in Frankreich fand der Vertrag keine allgemeine Zustimmung. Auf allen Seiten formierte sich die Opposition. Bundeskanzler Adenauer sah sich dem diplomatischen Druck der Vereinigten Staaten ausgesetzt, die ihm einen allerdings nebulösen Gegenvorschlag machten: Die Bundesrepublik könne an der multilateralen Atomstreitmacht (MLF) teilnehmen, die die Vereinigten Staaten im Rahmen der NATO zur Verteidigung Europas auf die Beine stellen wollten. Adenauer verfügte nicht mehr über genügend innenpolitischen Handlungsspielraum, um seinen Standpunkt durchzusetzen, als er Washington über die Natur des deutschfranzösischen Unterfangens beruhigte. Er mußte schließlich die Vorschläge der Mitglieder seines Kabinetts akzeptieren, dem Vertrag eine Resolution des Bundestages voranzustellen, in der die Treue der Bundesrepublik gegenüber der Allianz erneut beteuert wurde. Daraus entstand, inspiriert von Jean Monnet, der eine zu exklusive Achse Paris-Bonn als Hindernis auf dem Weg zu einer europäischen Integration ansah, die berühmte Präambel vom 16. Mai 1963. In ihr wurde festgehalten, daß der Vertrag weder die Beziehungen zwischen Europa und den Vereinigten Staaten noch die gemeinsame Verteidigung im Rahmen der NATO verändern würde. ${ }^{19}$ Es war im Grunde ein Unterwer-

18 Manfred STEINKÜHLER, Der deutsch-französische Vertrag von 1963: Entstehung, diplomatische Anwendung und politische Bedeutung in den Jahren von 1958 bis 1969, Berlin 2002.

19 Florence GAUZY, L'Allemagne, la France et l'OTAN: le temps des malentendus (19631964), in: CEHD, C2SD (Anm. 3), S. 27-39. 
fungsakt gegenüber den Vereinigten $\operatorname{Staaten}^{20}$ und der Triumph einer atlantischen Linie, die sich anschickte, in Bonn die Oberhand zu gewinnen. ${ }^{21}$

Einige Tage vor dem triumphalen Besuch von Präsident Kennedy im Sommer 1963 und seiner Rede in Berlin, die bei den Zuhörern einen Begeisterungssturm auslöste, kündigte de Gaulle an, die französische Flotte im Atlantik und im Ärmelkanal dem integrierten Kommando der NATO zu entziehen. Bei seinem Aufenthalt in Bonn im Juli fiel es ihm schwer, seine Verbitterung durch eine rednerische Pirouette zu maskieren, als er Verträge mit Rosen und jungen Mädchen verglich, die eben nur eine kurze Blütezeit hätten. $\mathrm{Zu}$ diesem Zeitpunkt hatte er dennoch nicht jegliche Hoffnung aufgegeben, sein großes Projekt einer europäischen Verteidigung innerhalb einer reformierten Allianz zu verwirklichen. Angespornt durch eine Bemerkung von Bundeskanzler Adenauer, umschrieb er die deutsch-französischen Beziehungen schließlich mit einem Rosengarten, der solange gedeihe, wie man ihn pflege. ${ }^{22}$ Wie im Vertrag vereinbart fanden im Laufe des Jahres 1963 mehrere Treffen zwischen den Verteidigungsministern und den Stabschefs statt: Der Ton war herzlich und die Gespräche zwischen den militärischen Verantwortlichen konstruktiv. Aber das reichte nicht aus, um die diplomatische Attacke der Vereinigten Staaten abzuwehren. Durch die Aufrechterhaltung von fast ständigen Kontakten behaupteten sie sich als einziger Gesprächspartner der Bundesrepublik in diesem Sommer 1963, in dem über das Dokument MC/100/1 der NATO diskutiert wurde, in dem die neue Nukleardoktrin der flexible response präzisiert wurde ("McNamara-Doktrin«). In ihr wurde nun im Falle einer sowjetischen Aggression den konventionellen Waffen eine bedeutendere Rolle zugewiesen und damit die Schwelle vor dem Einsatz von Nuklearwaffen angehoben. Aus amerikanischer Sicht würde die Schlacht so weit wie möglich im Osten stattfinden, um das deutsche Territorium zu schützen: Jedes Mitglied der NATO sollte einen Teil der Abwehr übernehmen. ${ }^{23}$ Trotz gemeinsamer Interessen - die Gefahr für beide, Westeuropa zu verlieren und seiner Zerstörung durch taktische Nuklearwaffen beizuwohnen - gelang es der Bundesrepublik und Frankreich nicht, eine gemeinsame Position zu MC/100/1 zu erarbeiten. Im Militärausschuß der NATO lehnte nur Frankreich, in der Person des Generals Charles Ailleret, dieses Dokument kategorisch ab. Er beharrte auf dem Konzept der französischen Abschreckung, das einen sofortigen Ein-

Reiner POMMERIN, L'attitude américaine face aux relations franco-allemandes, in: CEHD, C2SD (Anm. 3), S. 104.

21 Reiner MARCOWITZ, Option für Paris? Unionsparteien, SPD und Charles de Gaulle 1958 bis 1969, München 1996.

22 SOUTOU (Anm. 9), S. 256.

23 Maurice VAIISSE, La Grandeur. Politique étrangère du général de Gaulle 1958-1969, Paris 1998, S. 139-151. 
satz von Nuklearwaffen im Falle einer Bedrohung des nationalen Territoriums vorsah, aber noch ohne seine Integration in die Planung der NATO in Frage zu stellen. Bonn seinerseits suchte nach einer Kompromißlösung mit den amerikanischen Stellen. Diese Tendenz verstärkte sich mit dem Amtsantritt der neuen Regierung Erhard und ihres Außenministers Schröder im Herbst 1963, die in den gaullistischen Vorstellungen ein gefährliches Abenteuer sahen, das bestens dazu geeignet sei, Deutschland des segensreichen amerikanischen Schutzschildes zu berauben: Von nun an wurden die Anfänge der deutschfranzösischen Annäherung, die von de Gaulle und Adenauer skizziert wurden, zugunsten einer prinzipiellen Anpassung an die Nuklear- und Europapolitik der USA mit Füßen getreten, ${ }^{24}$ so daß einige von »Verrat « sprachen. ${ }^{25}$

Worauf ist dieser Umschwung zurückzuführen? Einerseits auf Unverständnis, ja sogar Verärgerung, die im Kabinett Erhard die gaullistische Politik des Beharrens auf nationaler Souveränität innerhalb der NATO und den europäischen Einrichtungen hervorrief. Andererseits auf die Überzeugung, daß Frankreich allein nicht in der Lage sei, im Falle einer Aggression eine ausreichende Sicherheitsgarantie für die Bundesrepublik zu gewährleisten. Dieser zweite Faktor war entscheidend. Die Möglichkeit einer deutsch-französischen strategischen Kooperation ist in Bonn niemals als eine denkbare Sicherheitsalternative betrachtet worden. Es ist auch nicht sicher, ob diese Möglichkeit in Paris stets sehr ernst genommen wurde. ${ }^{26}$ In diesem Zusammenhang ist die Episode vom 21. November 1963, dem Tag der ersten Unterhaltung zwischen de Gaulle und Erhard in Paris, besonders signifikant. Es gibt einen Streit zwischen Historikem und Zeitzeugen über die Tragweite des Engagements, das der General an diesem Tag einging. Welche Rolle sollte der französischen Force de frappe im Falle eines Angriffs der Sowjetunion auf Deutschland zukommen? Es trifft zu, daß das französische Angebot unklar formuliert war, da der General klugerweise sehr vage über das Ausmaß und die Art der Ausweitung des "Sanktuariums « blieb. Handelte es sich für Deutschland um eine geschenkte Garantie ohne die Möglichkeit der Teilnahme an der Ausarbeitung der französischen Nuklearpolitik oder um eine wirkliche Beteiligung an der Einsatzdoktrin der Waffen? Der Zweifel bestand fort, doch sprechen wir uns im Zweifel für den "Angeklagten« de Gaulle aus, der in den Jahren 19581963 tatsächlich an eine weitgehende deutsch-französische nukleare Zusammenarbeit gedacht $\mathrm{zu}$ haben schien, aber eben zu seinen Bedingungen. ${ }^{27}$ 
Die Monate, die diesem Gespräch folgten, brachten keine Änderung in den deutsch-französischen Beziehungen, die noch immer von deutlichen Spannungen gekennzeichnet waren. Frankreich warf der Bundesrepublik vor, nicht auf sein Angebot der strategischen Zusammenarbeit zu antworten, da man sich nicht ein für alle Mal zwischen Paris und Washington entscheiden und sich auch nicht eindeutig auf eine europäische und unabhängige Politik festlegen wolle. Die Regierung Erhard antwortete, daß Paris sie vor eine unmögliche Wahl stelle. Im Juli 1964 schien sich ein Status quo entwickelt zu haben. Das war just der Moment, den General de Gaulle wählte, um dem Élysée-Vertrag eine zweite Chance zu geben. Am 4. Juli 1964 unterbreitete er erneut sein Angebot zur politischen und militärischen Kooperation, um, so sagte er, um den deutsch-französischen Kern herum ein Gegengewicht gegen die amerikanische Macht in Europa zu bilden. Und er wiederholte gleichzeitig sein Angebot, die Force de frappe automatisch für die Verteidigung Europas einzusetzen. Verblüfft fragte Erhard, ob dies bedeute, daB Europa in diesem Fall über eine eigene Atomstreitmacht verfüge. In kurzer Zeit, so fuhr de Gaulle fort, werde Frankreich seine atomaren Einheiten in den Dienst der Verteidigung Europas stellen, bis dieses über eine autonome Streitmacht in den Händen einer politischen Union verfüge. Das Angebot kam völlig unverhofft: Erhard hörte es, verstand es aber nicht wirklich und verfiel wieder in das klassische Schema, indem er darauf hinwies, daß es die Priorität seiner Regierung bleibe, die Bedingungen für eine Teilnahme an der nuklearen Planung und Strategie im Rahmen des Atlantischen Bündnisses zu schaffen. Am Rande der offiziellen Gespräche wandte sich de Gaulle an Staatssekretär Carstens. Es war ein letzter Versuch, aber auch dieser blieb erfolglos. ${ }^{28} 1966$ bestätigte die Gründung der nuklearen Planungsgruppe bei der NATO als Ersatz für die Totgeburt der Multilateralen Atomstreitmacht die Bundesrepublik in ihrer Wahl, verstärkte ihre Atlantische Integration und entfernte sie noch mehr von Frankreich, das einer konzertierten Abschreckung auf europäischer Ebenen ablehnend gegenüberstand.

\section{Die Folgen des Vertrages}

Im Kontext der Jahre 1963-1966 konnte das kurz- und mittelfristige Ziel der Unterzeichner des Vertrages in den Fragen der Verteidigung nur das eine sein: zu einer gemeinsamen Position gegenüber der Ankündigung der McNamara- 
Doktrin der flexible response sowie der Reform der Allianz zu gelangen, die sowohl de Gaulle als auch Adenauer für notwendig hielten, um die Sicherheit Europas besser garantieren zu können. Nach der Affäre um die Präambel und die abweisende Haltung der Deutschen, zog der General für Frankreich die Konsequenzen aus der aus seiner Sicht negativen Bilanz: 1966 beorderte er die französischen Truppen aus den integrativen Strukturen der NATO zurück und schwang sich zum Meister einer nationalen Unabhängigkeitspolitik auf, deren Garant die Force de frappe war. Ab diesem Zeitpunkt gab es aufgrund der unterschiedlichen Ausgangspositionen und ihrer abweichenden strategischen Interessen keine wirklich gemeinsame Position mehr: hier Frankreich mit seinen Atomwaffen und einem großen Hinterland (die überseeischen Besitzungen), dort die Bundesrepublik, an der Grenze zum Ostblock, integriert in eine defensive Allianz und abhängig vom atomaren Schutz der Amerikaner. Der Kern des deutsch-französischen Problems in den Fragen der Verteidigung lag genau darin: in der Beziehung zur NATO und über die NATO zu den Vereinigten Staaten und insbesondere zu ihrer Atomwaffenpolitik. ${ }^{29}$ Untrennbar verbunden mit der gaullistischen Vision von nationaler Unabhängigkeit und einem souveränen Europa verschärfte sich das Problem noch, als Frankreich dem Club der Atommächte beitrat. Bonn war nun gezwungen, zwischen Washington und Paris zu wählen und entschied sich vernunftgeleitet, um so mehr, als die französische Alternative bis zum Schluß eine Grauzone enthielt, über die wir bereits gesprochen haben. Von nun an hatten sich die Bundesrepublik und Frankreich in strategischen Fragen nicht mehr viel zu sagen. Auch wenn der Dialog offiziell niemals wirklich abgebrochen wurde, war die deutschfranzösische militärische Zusammenarbeit in eine politisch-strategische Sackgasse geraten. Sie blieb dort fast 30 Jahre lang, durchlebte Höhen und Tiefen, die man mehr im Detail untersuchen könnte, aber die wir hier bewußt übergehen, da dies bereits anderswo geschehen ist. ${ }^{30} \mathrm{Da}$ es in diesen Jahren zu keinen gemeinsamen Doktrinen kommen konnte, muß also in strukturellen Inkompatibilitäten gesehen werden.

Verweisen wir dennoch kurz als Beispiel auf einen dieser plötzlichen Sprünge: das "Wiederaufleben « des Vertrages Anfang der 1980er Jahre auf Initiative von Bundeskanzler Helmut Schmidt und Präsident Giscard d'Estaing. Von den Zeitzeugen als ein beachtlicher Fortschritt bejubelt, hat der Versuch der Wiederbelebung eine Kettenreaktion ausgelöst: das Konzept der "Schicksalsgemeinschaft" zwischen diesen beiden Ländern und ein Übergang zu einer Aufweichung des französischen Verständnisses von Abschreckung; behauptung 1945-2000, Stuttgart, München 2001.

30 SOUTOU (Anm. 9). 
die Gründung der deutsch-französischen Brigade 1986, dem Embryo einer zukünftigen europäischen Streitmacht; die Schaffung einer zwischenstaatlichen Planungsgruppe, des deutsch-französischen Sicherheits- und Verteidigungsrates, der 1988 seine Tätigkeit aufnahm. Ohne den individuellen Wert eines jeden dieser Ereignisse mindern zu wollen, bleibt jedoch festzustellen, daß ihr Beitrag bei der Harmonisierung der strategischen Doktrinen eher gering war. Es handelte sich bestenfalls um eine Rückkehr zum Vertragstext.

Wie ging dies vor sich? Angesichts der Verschlechterung des internationalen Umfeldes, gekennzeichnet von der sowjetischen Intervention in Afghanistan (1979) und dem NATO-Doppelbeschluß, war man in Paris und Bonn über die amerikanische Haltung in den Verhandlungen über eine Begrenzung der strategischen Rüstungen zwischen der UdSSR und den USA (SALT) sowie über eine beiderseitige ausgewogene Truppenreduzierung (MBFR) beunruhigt, die zu einer Verringerung der nuklearen und konventionellen Waffen führen sollten. Wieder einmal waren es die Vereinigten Staaten, die indirekt ein Motiv für die deutsch-französische Annäherung lieferten. Mittlerweile durch eine persönliche Freundschaft verbunden entschlossen sich Bundeskanzler Helmut Schmidt und Valéry Giscard d'Estaing, die sich der Risiken für Europa im Falle günstiger Verhandlungen für die Sowjetunion bewußt waren, dem Vertrag von 1963 wieder neues Leben einzuhauchen und sprachen gemeinsam, im Juli 1980 in Baden-Baden, von der "Schicksalsgemeinschaft" zwischen Frankreich und Deutschland, der man einen Sinn geben müsse. Der westdeutsche Kanzler ging noch weiter, indem er vorschlug, auf dieser Basis eine wirkliche europäische Verteidigung zu schaffen, zu der jedes Mitglied einen eigenen Beitrag leisten sollte, anders gesagt hie $B$ das für Frankreich die Force de frappe. $^{31}$

Das war ein Schritt zu viel in Richtung auf ein Tabu-Thema, die französische Abschreckung, und Valéry Giscard d'Estaing präzisierte sogleich, daß es keineswegs eine atomare Garantie durch Ausweitung des "Sanktuariums" geben könne. Zwei Jahre später äußerte sich auch François Mitterrand, der mit Helmut Kohl den von ihren Vorgängern begonnenen Versuch einer Annäherung fortsetzte und Arbeitsstrukturen schuf, die 1988 zum deutschfranzösischen Sicherheits- und Verteidigungsrat wurden, in bezug auf die strategische Planung sehr klar. Die Angliederung der Bundesrepublik an die französische Nuklearstrategie stünde keineswegs zur Debatte, ebenso wenig eine Weitergabe von Nukleartechnologie an Deutschland noch ein irgend gearteter Beitrag Deutschlands zur Entwicklung der Force de frappe. ${ }^{32}$ Die Zweideutigkeit der deutsch-französischen Annäherung war offensichtlich, und

31 JARDIN, KimMEL (Anm. 14), S. 54.

32 Ministère des Affaires étrangères, Bulletin d'information, 26.10.1982. 
zu einem neuen "Strauß-Chaban-Abkommen « (1957) war der Weg noch weit. Was den deutsch-französischen Sicherheits- und Verteidigungsrat anging, dessen Organisation, Aufgaben und Funktionsweise in einem Zusatzprotokoll zum Élysée-Vertrag geregelt wurden, muß man feststellen, daß er über die institutionelle Einrichtung hinaus im Grunde nichts Neues brachte: "Er schafft keine deutsch-französische Verteidigungsdoktrin. Er verändert nichts an den von Frankreich gegebenen Garantien für die Sicherheit der Bundesrepublik. Er geht nicht auf die unterschiedliche Art der Beteiligung jedes der beiden Länder an der Atlantischen Allianz ein. Er enthält nicht ein einziges Projekt für die gemeinsame Rüstungsproduktion. Keines der beiden Länder verzichtet darauf, eine Verteidigungspolitik nach eigenem Gutdünken zu führen«, vermerkte ein französischer Abgeordneter während der Debatte zur Ratifizierung.

Es bleibt aber auch immer wieder zu betonen, daß die strategische Sackgasse, in der sich Deutschland und Frankreich im Laufe dieser langen Jahre befanden, keineswegs Erfolge im Bereich der deutsch-französischen Militärkooperation unterhalb der Suche nach gemeinsamen Doktrinen verhinderte. Der fast tägliche Kontakt zwischen den beiden Armeen, erleichtert durch den Verbleib von französischen Truppen in Deutschland bis 1994, der auf den nach dem Rückzug Frankreichs aus der militärischen Integration (1966) überarbeiteten Statuten beruhte, trug sehr viel dazu bei, ebenso wie die ständigen Bemühungen der Bundesrepublik, zwischen Frankreich und den integrierten Strukturen der NATO zu vermitteln. Es sind also die sekundären, aber nicht unbedingt zweitrangigen Aspekte des Vertrages, in denen Fortschritte zu beobachten waren: die Annäherung zwischen den Menschen, der Austausch von Personal und ganzen Einheiten, ${ }^{33}$ die Ausbildung, die Manöver und die gemeinsamen Übungen, wofür 1987 das Manöver »Kecker Spatz« das beste Beispiel bleibt, ${ }^{34}$ die logistische Unterstützung, die industrielle Zusammenarbeit im Rüstungssektor mit den Programmen Transall, Roland, Tiger, A-400... - so viele konkrete Umsetzungen, deren Auswirkungen schwer zu beziffern sind, solange es noch keine Arbeiten über diese spezifischen Themen gibt. Sie haben sicherlich nicht den erhofften Umfang erreicht, aber sie haben dennoch einen dauerhaften Effekt auf die militärische Praxis der beiden Länder gehabt und können gegebenenfalls auch die Bedingungen für eine zukünftige effiziente Zusammenarbeit schaffen. Bereits 1991 bemerkte General de Maizière zu

Manfred RosenberG, Die Annäherung der Menschen in den Streitkräften über eine Politik des Personalaustausches und der systematischen Zusammenarbeit, in: CEHD, C2SD (Anm. 3), S. 237-242.

34 Benoit Rossignol, Apprendre l'un et l'autre: l'École de guerre navale et la coopération navale franco-allemande, in: CEHD, C2SD (Anm. 3), S. 243-280. 
dieser Frage in seinem unnachahmlichen Stil, einer Mischung aus Diplomatie, Realismus und gutem Willen:

Eine weitere substantielle Vertiefung der deutsch-französischen militärischen Zusammenarbeit setzt eine Überwindung der nach wie vor bestehenden grundsätzlichen Auffassungsunterschiede im sicherheitspolitischen und strategischen Bereich voraus. Unterhalb dieser Ebene aber ist der vorhandene Spielraum noch nicht ganz ausgeschöpft. Denkbar bleiben z.B. noch eine Ausweitung der personellen Beziehungen, eine Annäherung der taktischen Doktrinen und die Verbesserung der Interoperabilität im logistischen und im Rüstungsbereich. $^{35}$

Mit dem Ende des Kalten Krieges bahnte sich seit dem Vertrag von Maastricht die Idee einer eigenen europäischen Verteidigung um einen deutschfranzösischen militärischen Kern herum in den Köpfen und in den Texten langsam ihre Bahn. Sicherlich, sie hauchte der deutsch-französischen Zusammenarbeit neues Leben ein, indem sie den bilateralen Rahmen überwand, ohne jedoch die Hypotheken aus der Vergangenheit aufzuheben. Der Bezugsrahmen blieb die Atlantische Allianz und, im Rahmen der NATO, die amerikanische Verteidigungspolitik, der gegenüber, wie man weiß, Deutschland und Frankreich keine gemeinsame Position vertreten. Dort, wo Paris lange Zeit für eine wirkliche europäische Eigenständigkeit in Sicherheit und Verteidigung eingetreten war, ausgestattet mit einem bewaffneten Arm im Rahmen einer reformierten WEU, optierte die Bundesrepublik eher für einen europäischen Pfeiler im Rahmen des Atlantischen Bündnisses. Die NATO, die Vereinigten Staaten und die atomare Abschreckung stellten damit beständige Zankäpfel zwischen den beiden Ländern dar. Hinzu kam seit Ende der 1990er Jahre, die nach dem Ende des Kalten Krieges von neuen Friedenshoffnungen geprägt waren, ein neues Element: die unterschiedliche Bedeutung, die Deutschland und Frankreich der militärischen Stärke in der Definition der Europäischen Sicherheitsund Verteidigungspolitik (ESVP) einzuräumen gewillt scheinen. Denn Berlin versteht unter Sicherheit zunehmend nicht mehr nur militärische Aspekte. In diesem Zusammenhang ist es recht symptomatisch, daß sich beide Länder Mitte der 1990er Jahre auf eine große Umgestaltung ihrer militärischen Apparate eingelassen haben, ohne jegliche bilaterale Konsultation. Als Folge der Professionalisierung der französischen Streitkräfte 1996, die von großen Anstrengungen zur Modernisierung der Bewaffnung begleitet wurde, gibt es nunmehr zwei unterschiedliche Armeearten, unter strikten budgetären Auflagen, insbesondere in Deutschland. In aller Deutlichkeit ist zu erkennen, da $B$ die verantwortlichen Politiker der beiden Länder auch nicht an dieselbe Art von Missionen denken, sofern die rot-grüne Koalition, die in Deutschland seit 1998 an der Macht ist, überhaupt irgendeine militärische Ambition außerhalb 
der Landesverteidigung und der Petersberger Aufgaben verspürt. Das Budget, das man dem Verteidigungssektor zugesteht (weniger als 1,5\% des BIP), läßt daran Zweifel aufkommen.

Tatsächlich keimte erst nach dem 11. September 2001 und dem radikalen Wechsel der amerikanischen Strategie im Verlauf der Operationen in Afghanistan wieder die Hoffnung auf eine zweite Blüte des Élysée-Vertrages auf, indem auf Konsultationen jetzt auch Aktionen folgen. In einer auf 19 und bald 26 Mitglieder $^{36}$ erweiterten Atlantischen Allianz, deren internationaler Einfluß durch die Bildung multilateraler Ad hoc-Streitkräfte unter amerikanischer Ägide wie in Afghanistan und dann im Irak-Krieg notwendigerweise geschwächt wird, könnten sich die deutsch-französischen Erfahrungen auf politisch-strategischer Ebene, aber auch im Bereich der Ausbildung und in der Rüstung, kurz für die Interoperabilität der Streitkräfte als ein entscheidender militärischer Vorteil für die Vermeidung und Bewältigung von zukünftigen Konflikten herausstellen. Wenn sich diese Annahme bestätigt, stünde nicht mehr in erster Linie der strategische Aspekt des Vertrages im Vordergrund, dieser merkwürdige Ehrgeiz, eine gemeinsame Position zwischen Ländern mit unterschiedlichen strategischen Interessen zu entwickeln, sondern der traditionelle militärische Aspekt: der Austausch von Mensch, Material und Technologie, der auch vom Vertrag vorgesehen war und der, von Fall zu Fall, erlauben würde, auf ganz pragmatische Weise eine sehr weitgehende Kooperation anzustreben, jedes Mal wenn sich die Notwendigkeit dazu ergibt.

\section{Schlußfolgerung}

Was bleibt vom Élysée-Vertrag im Bereich der Verteidigung noch übrig? Vordergründig nur wenige Dinge: Routinetreffen, ein ritualisierter Dialog, standardisierte Kommunikationsgewohnheiten, Absichtserklärungen, akzeptiertes Schweigen, ein Gipfeltreffen, das manchmal unter zuviel Formalismus erstickt, eine Vision von einem auf gemeinsamer Basis noch aufzubauenden Europa der Verteidigung, aber im Grunde weder eine deutsch-französische Doktrin noch eine reelle Einheit der gemeinsamen operationellen Verteidigung. 40 Jahre nach der Unterzeichnung des Élysée-Vertrages muß man feststellen, $\mathrm{da} ß$ die deutsch-französische Verteidigung keine wirklich greifbare militärische Umsetzung erfahren hat, abgesehen vom Eurocorps, dessen Bestand und Einsatzmöglichkeiten gleichwohl von der NATO abhängen. Man 


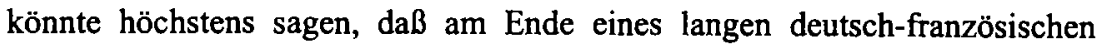
Dialoges in Verteidigungsfragen, wenn man sich auf das jeweilige Verhalten während des Beginns der militärischen Operation in Afghanistan (2001) und dann in der Irakkrise (Sommer 2002) stützt, Deutschland ein wenig französischer und Frankreich ein wenig deutscher geworden ist. Man kann sich dazu gratulieren oder darüber traurig sein, je nachdem, wie man die strategischen Doktrinen eines jeden versteht und je nach der Definition, die man einer gemeinsamen Zukunft geben möchte. Wer kann wissen, ob das Ergebnis den Ambitionen der Architekten des Vertrages entspricht.

Wovon hängt diese Diskrepanz zwischen Intention und Realisation ab? Der Élysée-Vertrag definierte im Verteidigungsbereich bislang unerreichte Kooperationsziele. Waren diese Ziele unrealisierbar? Unbestreitbar beschleicht jeden, der den Text buchstabengetreu liest, das Gefühl, daß seine Architekten sich eitlen Hoffnungen hingaben (die Anpassung der taktischen und strategischen Doktrinen) und durch Unterlassung (die weiter bestehende Ungenauigkeit über das Ausmaß dieser neuen Kooperation - sollte sie auch den Atomsektor umfassen oder nicht?) Fehler begingen. Aber sollte man dem Vertrag Buchstabe für Buchstabe folgen? Sollte man ihn nicht lieber wieder in seinen historischen Zusammenhang stellen und als das betrachten, was er vor allem ist: ein feierlicher politischer Akt, der bis in seine Improvisationen und seinen Enthusiasmus hinein die Male der Krisen trug, die seiner Unterzeichnung vorausgingen. In seiner strategischen Dimension war der deutsch-französische Vertrag von 1963 ein in Krisenzeiten geborener Vertrag, der dazu gedacht war, Krisenzeiten zu bewältigen. Aus strategischer Perspektive kann man sich wohl kaum vorstellen, wie langfristig Doktrinen anzupassen sind, die auf gegensätzlichen Interessen basieren, geprägt durch eine grundsätzliche Asymmetrie der geopolitischen Lage der beiden Unterzeichner. De Gaulle und Adenauer waren viel zu pragmatisch, um das nicht zu bemerken. Hingegen ist es durchaus möglich, gemeinsame Konfliktlösungsstrategien zu entwickeln, wenn die Bedingungen dazu geschaffen sind. So könnte, wenn auch nicht der Buchstabe, so doch der Geist des Vertrages lauten.

Haben die Ziele im Bereich der Verteidigung durch einen manchmal fehlenden politischen Willen auf der anderen Seite des Rheins gelitten? Zweifellos in einigen ganz bestimmten Fällen. Aber über die ganze Periode betrachtet, haben die Regierungen in Bonn und Paris mehrere schwere Krisen überwunden, ohne jemals die Notwendigkeit des deutsch-französischen Bandes in Frage zu stellen. Die Akteure haben in ihrer Mehrheit viel zu seinem Fortbestehen beigetragen, und sei es nur durch herzliche und regelmäßige Beziehungen untereinander. War es also das internationale Umfeld, das während des Kalten Krieges das Entstehen einer gemeinsamen Position zwischen zwei Ländem verhindert hat, deren strategische Interessen offensichtlich verschieden waren und es auch bleiben sollten, falls nicht einer von ihnen auf die eigenen ver- 
zichten würde, um sich auf ein recht unsicheres Abenteuer einzulassen? Alle Faktoren haben, Mal für Mal, dazu beigetragen, aber der Kern der Frage liegt möglicherweise woanders.

Wenn man genauer hinsieht, ist die Bilanz nicht so enttäuschend wie sie scheint. 40 Jahre deutsch-französische Kooperation, sowohl auf politischstrategischem als auch im strengeren Sinne auf militärischem Sektor, haben zwischen Deutschland und Frankreich im Bereich der Verteidigung ein Netz von soliden Beziehungen geknüpft, das auf der besseren Kenntnis des anderen basiert, auf einer Tradition des Austausches von Personal und gemeinsamen Übungen, einer gemeinsamen Arbeitsroutine im Bereich der Rüstung, einer Gewöhnung an die fremden Kommandomethoden - so viele Erfahrungen also, die sich, auch wenn sie noch nicht völlig ausgeschöpft sind, im gegebenen Augenblick als entscheidend in einem internationalen Kontext herausstellen könnten, der von den nationalen Streitkräften immer mehr die Fähigkeit zur Interoperabilität erfordert. Der Élysée-Vertrag war Produkt und Opfer des Kalten Krieges, der seine Unterzeichner vor eine Quadratur des Kreises stellte, doch könnte er heute seinen militärischen Wert in einer anderen strategischen Konfiguration unter Beweis stellen.

(Übersetzung: Birgit Schöne-Martens) 
